\title{
Spectrum of Soft Computing Risk Assessment Scheme for Hypertension
}

\author{
Pankaj Srivastava \\ Department of Mathematics \\ Motilal Nehru National Institute of Technology \\ Allahabad, U.P., India, 211004
}

\author{
Amit Srivastava \\ Department of Applied Mechanics \\ Motilal Nehru National Institute of Technology \\ Allahabad, U.P., India, 211004
}

\begin{abstract}
The present paper deals with risk assessment scheme for Hypertension. It is believed that hypertension comes in to picture due to complex interaction of genetic, environmental and demographical features and nowadays it is a leading health problem in information technology world. In order to manage hypertension risk factor, we have made an attempt to design user friendly, intelligent and effective diagnostic system by making use of soft computing tools.
\end{abstract}

\section{Keywords}

Hypertension, CHD, FES, Risk assessment

\section{INTRODUCTION}

Hypertension is being considered as a leading silent killer disease throughout world now days. Medical experts are of the view that hypertension risk is root cause for raising the risk of chronic diseases such as kidney failure, heart disease, diabetes and cancer. The world health report published in 2002 recognized hypertension as the third ranked factor for disability adjusted life years. Recent analysis has predicted that more than 1.56 billon people will be living with hypertension worldwide by the year 2025 . As per available data, it has been confirmed that hypertension is responsible for $13 \%$ of death worldwide. P. Degoulet et al [1] described that High blood pressure or hypertension is a condition that occurs when the pressure in our arteries is consistently above the normal range. Blood pressure is the force of blood pushing against the wall of the arteries. Szolvits et al [2] viewed that medical researchers cannot precisely characterize how diseases alter the normal functioning of the body. The unpredictability and complexity features of hypertension force physicians sometimes to make decision on their intuition. All of these complexities in medical practice make traditional mathematical approach of analysis insufficient. Hobbs and Boyles [3] pointed out that complications of hypertension could lead to stroke or heart failure. Such complications may be caused by improper diagnosis and or improper management of the disease, due to inaccessibility of experienced medical practioners at all times. Fuzzy theory plays important role in such situations. X.Y. Djam et al [4] suggested that Fuzzy systems are excellent in handling ambiguous and imprecise information prevalent in medical diagnosis.

The literature available makes clear that different types of Artificial intelligence systems have already been designed for the diagnosis of hypertension. Riccardo P. et al [5] had proposed a Neural Network Expert System for Diagnosing and Treating Hypertension. Further Sylevie Charbonnier et al
[6] proposed the statistical and fuzzy models of Ambulatory systolic blood pressure for hypertension diagnosis. Novruz Allahverdi et al [7] proposed a fuzzy expert system to determination of coronary heart disease risk (CHD) of patient for next ten years. D.Pandey et al. [8] proposed a rule based system for cardiac analysis in which model developed on ECG based analysis. Pankaj Srivastava and Amit Srivastava [9] proposed a Fuzzy Expert System to determine coronary heart disease (CHD) risk of patients in India. In order to measure risk factor for hypertension, significant and user friendly fuzzy system has not been developed so far.

The present paper is focused on the design and development of fuzzy model to detect and diagnose hypertension risk factor using Age, BMI, blood pressure, heart rate, smoking, exercise, cholesterol level, triglyceride as input variables.

\section{METHODOLOGY}

\subsection{Fuzzy Expert System}

Chen and Chen [10] were of the view that Soft Computing is a computational method that is tolerant to sub-optimality, impreciseness, vagueness and thus giving quick, simple and sufficient good solutions. For complex systems, fuzzy tools is quite suitable because of its tolerance to some imprecision. Nalayini and Wahida [11] were of the view that most of the cardiac diseases are characterized by varied degrees of intricacy and the conventional procedures are not capable of dealing with these intricacies very efficiently. In the present study, the inputs consist of age, SBP, DBP, BMI, heart rate, LDL, HDL, triglyceride, smoking and exercise, while the output is the risk of hypertension (\%).

\subsection{Input Variables:}

a. Blood Pressure: In this field we use systolic BP (SBP) and diastolic BP (DBP). The input variables for SBP and DBP were classified in to seven fuzzy sets. Membership function of 'Normal' is ZMF and for 'Very high' sets SMF is used. Membership function of 'Above normal', 'moderate', 'above moderate', 'little high' 'High' sets are triangular.

\begin{tabular}{lll}
\hline & $\begin{array}{l}\text { Systolic BP in } \\
\mathbf{m m ~ H g}\end{array}$ & $\begin{array}{l}\text { Diastolic BP in } \\
\mathbf{m m ~ H g}\end{array}$ \\
\hline Normal & $<120$ & $<80$ \\
Above Normal & $120-130$ & $80-85$ \\
Moderate & $130-140$ & $85-90$ \\
$\begin{array}{l}\text { Above } \\
\text { Moderate }\end{array}$ & $140-150$ & $90-95$ \\
Little High & $150-160$ & $95-100$ \\
High & $160-170$ & $100-110$ \\
\hline
\end{tabular}




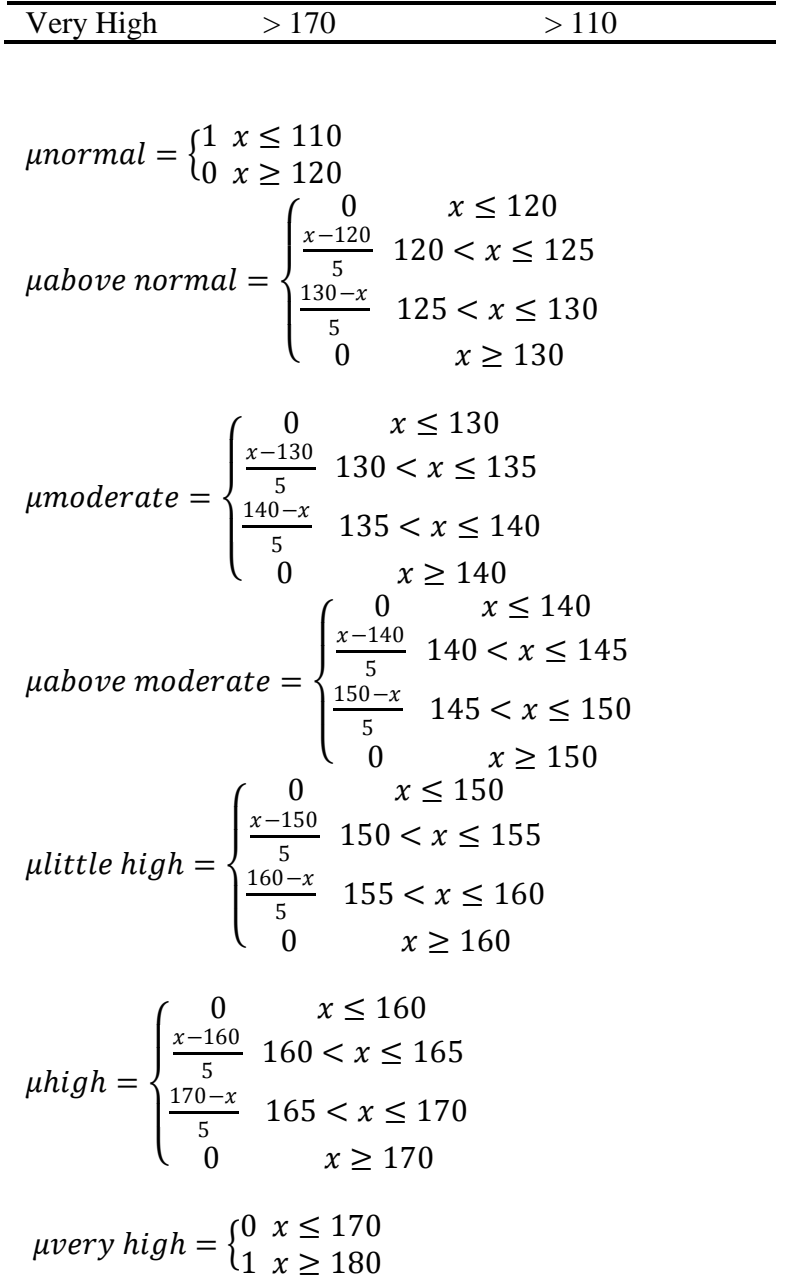

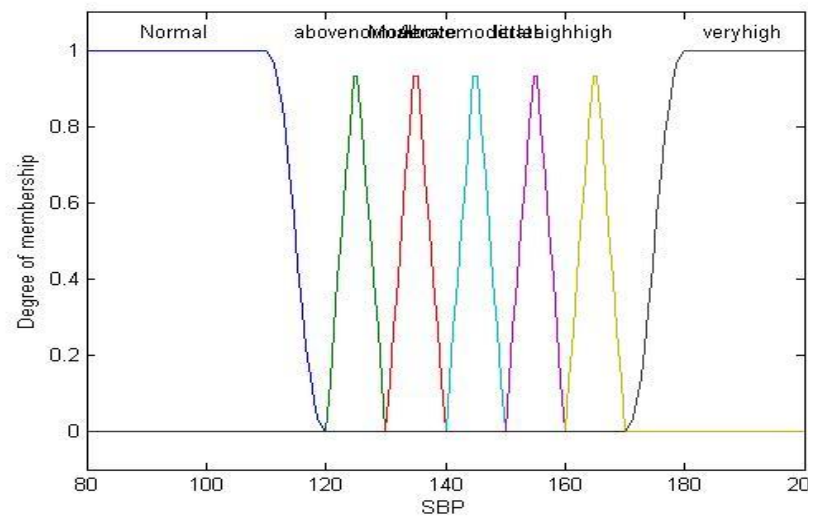

Figure1: Linguistic variables and membership function of Input variables 'SBP'

$$
\begin{aligned}
& \text { unormal }= \begin{cases}1 & x \leq 70 \\
0 & x \geq 80\end{cases}
\end{aligned}
$$

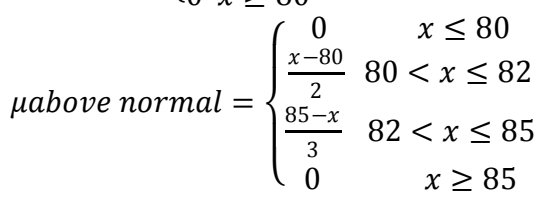

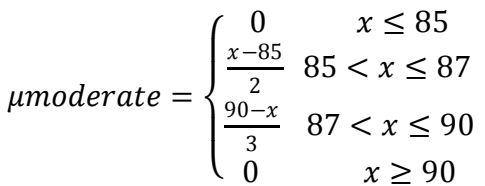

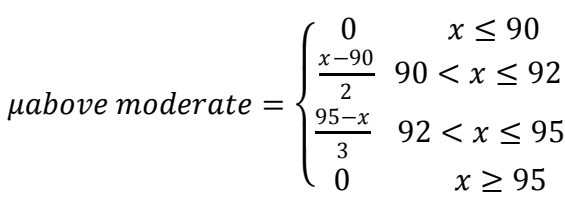$$
\text { plittle high }=\left\{\begin{array}{cc}
0 & x \leq 95 \\
\frac{x-95}{2} & 95<x \leq 97 \\
\frac{100-x}{3} & 97<x \leq 100 \\
0 & x \geq 100
\end{array}\right.
$$$$
\text { uhigh }=\left\{\begin{array}{cc}
0 & x \leq 100 \\
\frac{x-100}{5} & 100<x \leq 105 \\
\frac{110-x}{5} & 105<x \leq 110 \\
0 & x \geq 110
\end{array}\right.
$$$$
\text { uvery high }= \begin{cases}0 & x \leq 110 \\ 1 & x \geq 120\end{cases}
$$

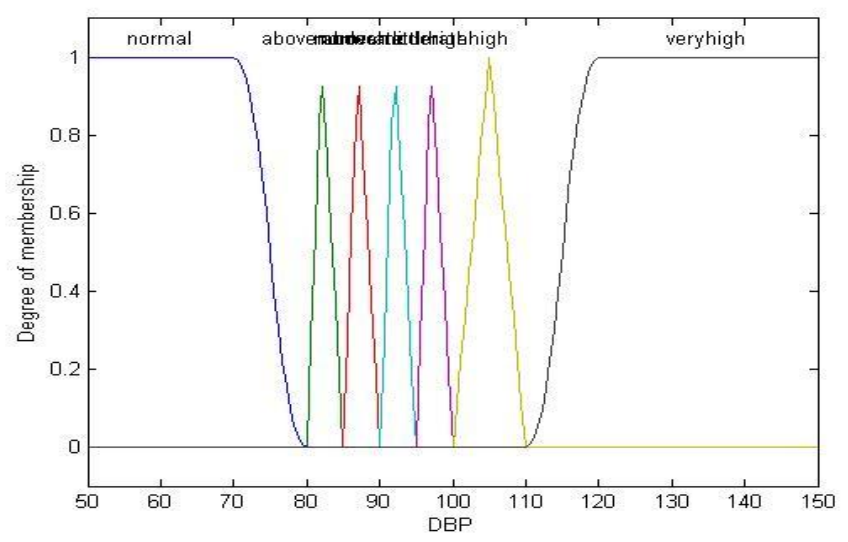

Figure2: Linguistic variables and membership function of Input variables 'DBP'.

b. Cholesterol: Cholesterol has been identified as one of the main risk factor for myocardial infarction. If Total Cholesterol level is too high or too low then further measurement of low density lipoprotein(LDL) cholesterol and High density lipoprotein (HDL) cholesterol are required. HDL Cholesterol level has been classified in four fuzzy sets (very high, high, nearly normal, normal). These fuzzy sets have been shown in table. Membership functions of very high, high, nearly normal sets are trapezoidal and for normal, SMF is used. LDL Cholesterol level has been classified in five fuzzy sets (optimal, above optimal, borderline high, high, very high). These fuzzy sets have been shown in table. Membership functions of normal, borderline, high sets were trapezoidal. For very high, SMF is used. 


\begin{tabular}{|c|c|c|c|}
\hline \multicolumn{4}{|c|}{ Cholesterol(mg/dL) } \\
\hline LDL & & HDL & \\
\hline Optimal & $0-100$ & Very high & $0-30$ \\
\hline Above Optimal & $100-130$ & & \\
\hline Borderline High & $130-160$ & High & $30-50$ \\
\hline High & $160-190$ & Nearly Normal & $50-60$ \\
\hline Very High & $\geq 190$ & Normal & $\geq 60$ \\
\hline
\end{tabular}

Hoptimal $=\left\{\begin{array}{lr}1 & x \leq 70 \\ 0 & x \geq 100\end{array}\right.$

Rabove optimal $=\left\{\begin{array}{cc}0 & x \leq 100 \\ \frac{x-100}{10} & 100<x \leq 110 \\ 1 & 110 \leq x \leq 120 \\ \frac{130-x}{10} & 120<x \leq 130 \\ 0 & x \geq 130\end{array}\right.$

$\mu$ borderline high $=\left\{\begin{array}{cc}0 & x \leq 130 \\ \frac{x-130}{10} & 130<x \leq 140 \\ 1 & 140 \leq x \leq 150 \\ \frac{160-x}{10} & 150<x \leq 160 \\ 0 & x \geq 160\end{array}\right.$

uhigh $=\left\{\begin{array}{cc}0 & x \leq 160 \\ \frac{x-160}{10} & 160<x \leq 170 \\ 1 & 170 \leq x \leq 180 \\ \frac{190-x}{10} & 180<x \leq 190 \\ 0 & x \geq 190\end{array}\right.$

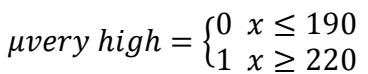

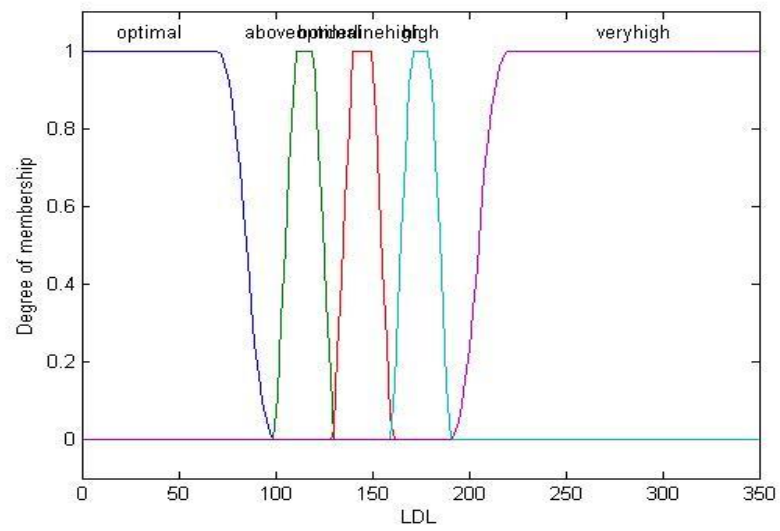

Figure3: Linguistic variables and membership function of Input variables 'LDL'

uvery high $=\left\{\begin{array}{cc}0 & x \leq 0 \\ \frac{x-0}{10} & 0<x \leq 10 \\ 1 & 10<x \leq 20 \\ \frac{30-x}{10} & 20<x \leq 30 \\ 0 & x \geq 30\end{array}\right.$

$$
\begin{aligned}
& \text { uhigh }=\left\{\begin{array}{cc}
0 & x \leq 30 \\
\frac{x-30}{5} & 30<x \leq 35 \\
1 & 35<x \leq 45 \\
\frac{50-x}{5} & 45<x \leq 50 \\
0 & x \geq 50
\end{array}\right. \\
& \text { 4nearly normal }=\left\{\begin{array}{cc}
0 & x \leq 50 \\
\frac{x-50}{3} & 50<x \leq 53 \\
1 & 53<x \leq 57 \\
\frac{60-x}{3} & 57<x \leq 60 \\
0 & x \geq 60
\end{array}\right.
\end{aligned}
$$$$
\text { unormal }= \begin{cases}0 & x \leq 60 \\ 1 & x \geq 70\end{cases}
$$

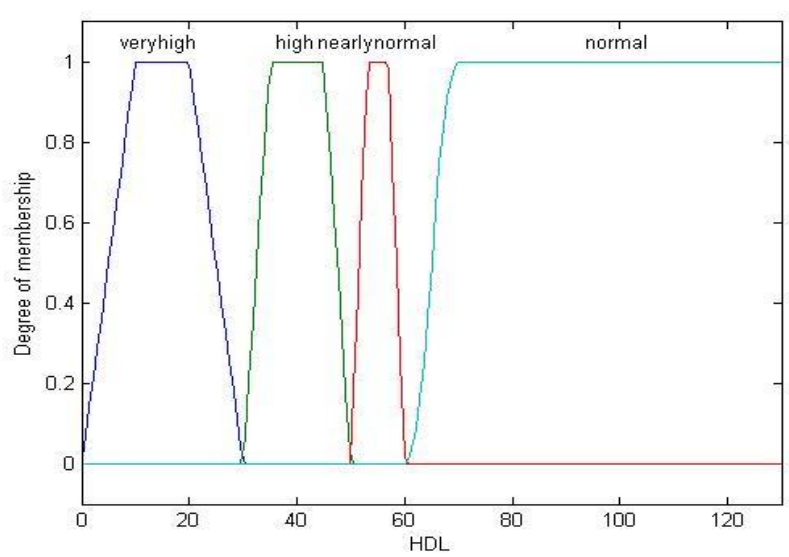

Figure 4: Linguistic variables and membership function of Input variables 'HDL'.

c. Age: This input field is classified in six fuzzy sets (young, Adult, Midaged, Aged, old, very old). The fuzzy sets with their range given in table. Membership function of young is $\mathrm{ZMF}$ and for very old, it is SMF. Membership functions of other's are trapezoidal.

\begin{tabular}{lc}
\hline & Age (in years) \\
Young & $<28$ \\
Adult & $25-48$ \\
Midaged & $45-60$ \\
Aged & $58-72$ \\
Old & $70-86$ \\
Very Old & $>82$ \\
\hline
\end{tabular}

$$
\text { pyoung }=\left\{\begin{array}{cc}
1 & x \leq 0 \\
0 & x \geq 28
\end{array} \text { uvery old }= \begin{cases}0 & x \leq 82 \\
1 & x \geq 90\end{cases}\right.
$$

padult $=\left\{\begin{array}{cc}0 & x<25 \\ \frac{x-25}{5} & 25 \leq x \leq 30 \\ 1 & 30 \leq x \leq 40 \\ \frac{48-x}{8} & 40 \leq x \leq 48 \\ 0 & x \geq 48\end{array}\right.$ 
нmidaged $=\left\{\begin{array}{cc}\frac{x-45}{5} & 45 \leq x \leq 50 \\ 1 & 50 \leq x \leq 56 \\ \frac{60-x}{8} & 56 \leq x \leq 60 \\ 0 & x \geq 60\end{array}\right.$

paged $=\left\{\begin{array}{cc}0 & x<58 \\ \frac{x-58}{4} & 58 \leq x \leq 62 \\ 1 & 62 \leq x \leq 66 \\ \frac{72-x}{6} & 66 \leq x \leq 72 \\ 0 & x \geq 72\end{array}\right.$

rold $=\left\{\begin{array}{cc}0 & x<70 \\ \frac{x-70}{4} & 70 \leq x \leq 74 \\ 1 & 74 \leq x \leq 78 \\ \frac{86-x}{8} & 78 \leq x \leq 86 \\ 0 & x \geq 86\end{array}\right.$

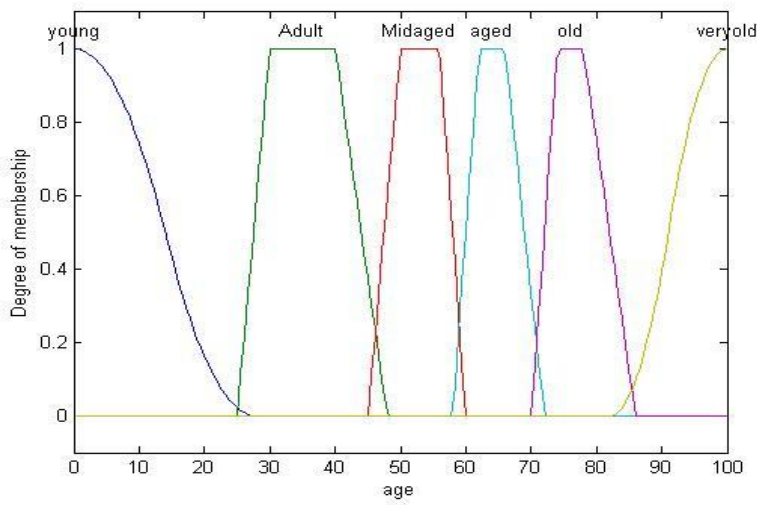

Figure5: Linguistic variables and membership function of Input variable 'Age'.

d. BMI- Body mass index is defined as the individual's body weight divided by square of his or her height. The formula used in medicine produce a unit of measure of $\mathrm{kg} / \mathrm{m}^{2}$. This input field is classified in four fuzzy sets. The fuzzy sets with their range are shown in table. Fuzzy sets are 'low (underweight)', 'medium (healthy weight)', 'high (slightly overweight)' and 'very high (overweight)' sets.

\begin{tabular}{lc}
\hline & Body mass index $\left(\mathbf{k g} / \mathbf{m}^{2}\right)$ \\
Low & $10-18$ \\
Medium & $15-26$ \\
High & $25-34$ \\
Very high & $32-40$ \\
\hline
\end{tabular}

plow $=\left\{\begin{array}{ll}1 & x \leq 10 \\ 0 & x \geq 18\end{array}\right.$ pvery high $= \begin{cases}0 & x \leq 32 \\ 1 & x \geq 40\end{cases}$

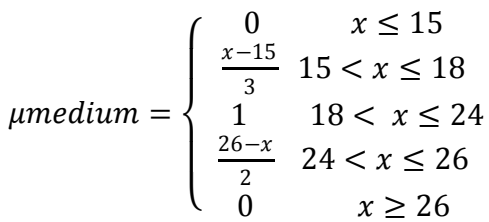

$$
\text { uhigh }=\left\{\begin{array}{cc}
0 & x \leq 25 \\
\frac{x-25}{2} & 25<x \leq 27 \\
1 & 27<x \leq 30 \\
\frac{34-x}{2} & 30<x \leq 34 \\
0 & x \geq 34
\end{array}\right.
$$

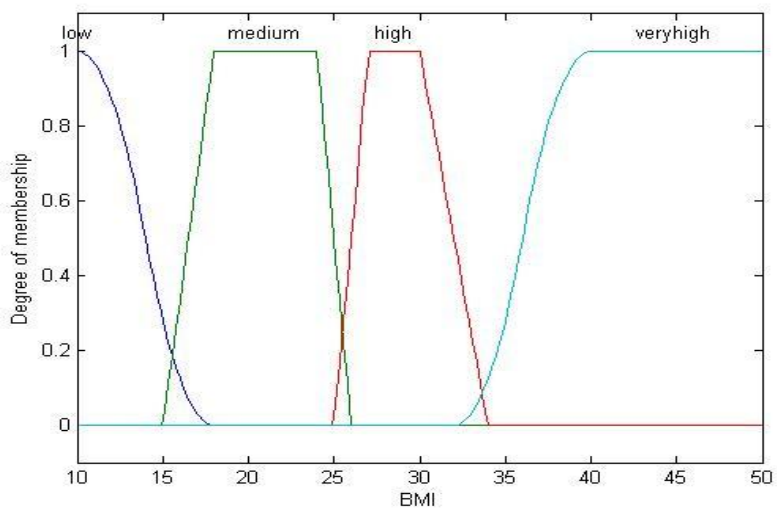

Figure6: Linguistic variables and membership function of Input variable 'BMI'.

e. Heart Rate- The value of this input field is heart rate of man during twenty four hours. By increasing of age in man, maximum of heart rate in twenty four hours decreases. In this field, we have four linguistic variables (low, normal, high, very high). The fuzzy sets with their range are shown in table.

\begin{tabular}{lc}
\hline & Heart Rate(beats/min) \\
\hline Low & $50-62$ \\
Normal & $60-80$ \\
High & $78-105$ \\
Very high & $100-120$ \\
\hline
\end{tabular}

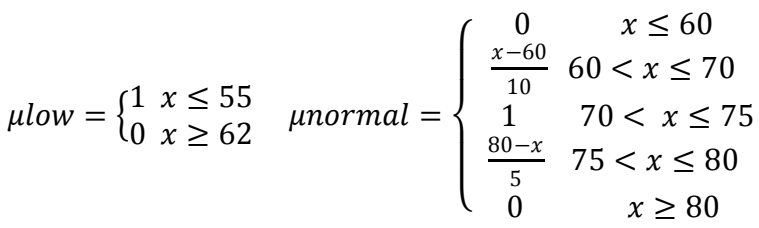

whigh $=\left\{\begin{array}{cc}0 & x \leq 78 \\ \frac{x-78}{8} & 78<x \leq 86 \\ 1 & 86<x \leq 95 \\ \frac{105-x}{10} & 95<x \leq 105 \\ 0 & x \geq 105\end{array}\right.$

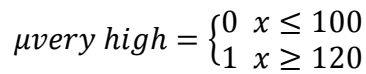




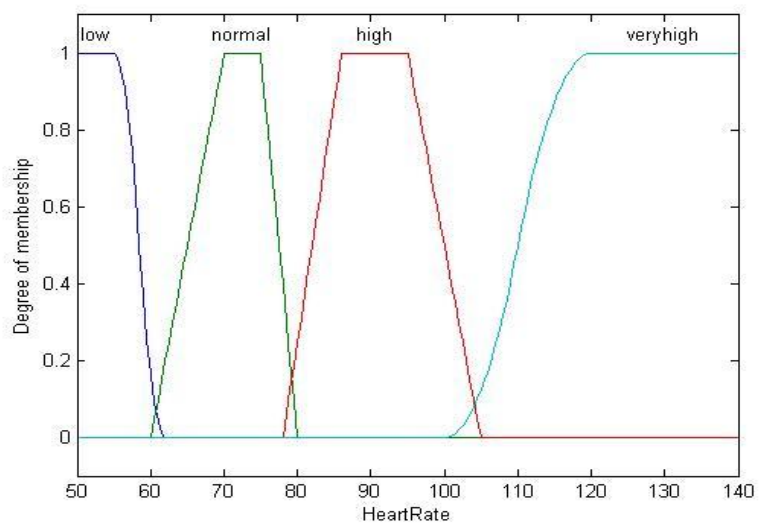

Figure7: Linguistic variables and membership function of Input variable 'Heart Rate'.

f. Triglyceride- Triglycerides are lipids, or fats, found in our bloodstream. Triglycerides play a major role in heart disease, heart attacks and strokes. The higher value of triglycerides just might be one cause of high blood pressure or hypertension. It is believed that a high level of triglycerides may contribute to atherosclerosis, which is the thickening or hardening of the arteries. Atherosclerosis will lead to high blood pressure. This input field is classified in four fuzzy sets. The fuzzy sets with their range are shown in table.

\begin{tabular}{lc}
\hline & Triglyceride $(\mathbf{m g} / \mathbf{d L})$ \\
\hline Normal & $<150$ \\
Borderline high & $150-200$ \\
High & $200-500$ \\
Very high & $\geq 500$ \\
\hline
\end{tabular}

Hnormal $=\left\{\begin{array}{lr}1 & x \leq 0 \\ 0 & x \geq 150\end{array}\right.$

?borderline $=\left\{\begin{array}{cc}\frac{x-150}{15} & 150<x \leq 165 \\ 1 & 165 \leq x \leq 185 \\ \frac{200-x}{15} & 185<x \leq 200 \\ 0 & x \geq 200\end{array}\right.$

phigh $=\left\{\begin{array}{cc}0 & x \leq 200 \\ \frac{x-200}{100} & 200<x \leq 300 \\ 1 & 300 \leq x \leq 400 \\ \frac{500-x}{100} & 400<x \leq 500 \\ 0 & x \geq 500\end{array}\right.$

pvery high $= \begin{cases}0 & x \leq 500 \\ 1 & x \geq 600\end{cases}$

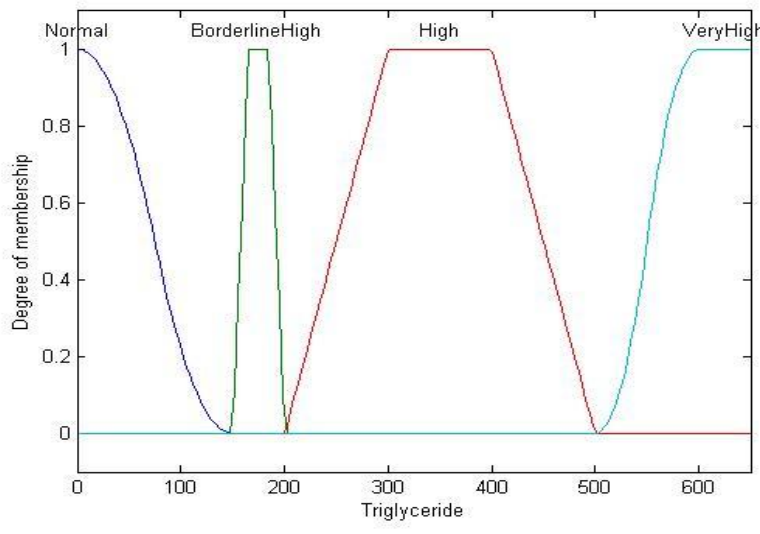

Figure8: Linguistic variables and membership function of Input variable 'Triglyceride'.

g. Exercise: This input field is classified in four fuzzy sets. We have considered moderate exercise as most of the people doing moderate exercising during daily life. The fuzzy sets with their range are shown in table. If person is not doing exercise then input value is zero.

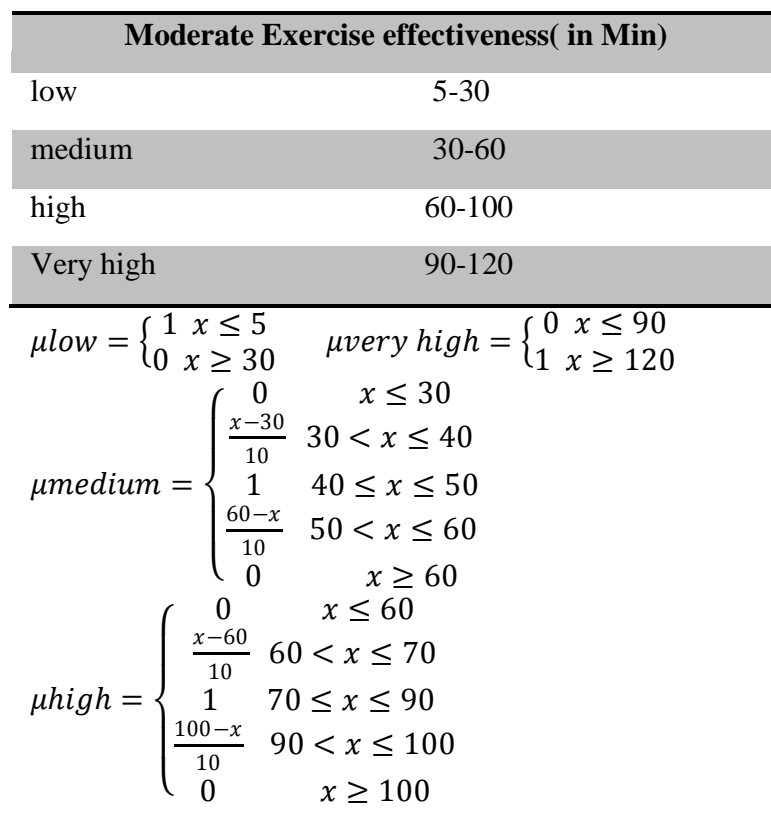

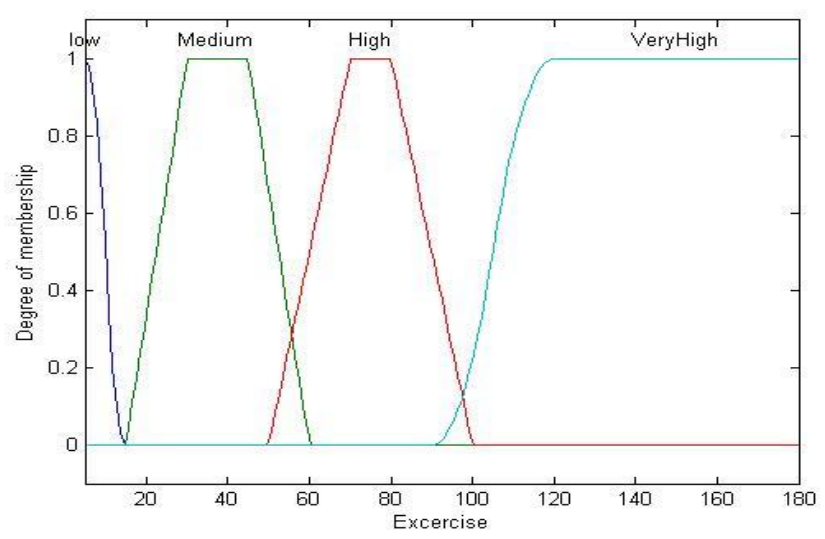

Figure9: Linguistic variables and membership function of 'Moderate Exercise'. 
h. Smoking: This input field is classified in four fuzzy sets. The fuzzy sets with their range are shown in table. If person is not doing smoking then input value is zero.

\begin{tabular}{|c|c|c|}
\hline \multicolumn{3}{|c|}{ Smoking } \\
\hline Low & $5-10 \mathrm{ci}$ & garettes \\
\hline Medium & $8-20 \mathrm{ci}$ & garettes \\
\hline High & $18-30 c$ & garettes \\
\hline Very high & $28-35$ & igarettes \\
\hline low $=\left\{\begin{array}{cc}1 & x \leq 5 \\
0 & x \geq 10\end{array}\right.$ & $\mu$ medium $=$ & $\begin{array}{cc}0 & x \leq 8 \\
\frac{x-8}{6} & 8<x \leq 14 \\
\frac{20-x}{6} & 14<x \leq 20 \\
0 & x \geq 20\end{array}$ \\
\hline
\end{tabular}

uhigh $=\left\{\begin{array}{cc}0 & x \leq 18 \\ \frac{x-18}{6} & 18<x \leq 24 \\ \frac{30-x}{6} & 24<x \leq 30 \\ 0 & x \geq 30\end{array}\right.$ Hvery high $= \begin{cases}0 & x \leq 28 \\ 1 & x \geq 35\end{cases}$

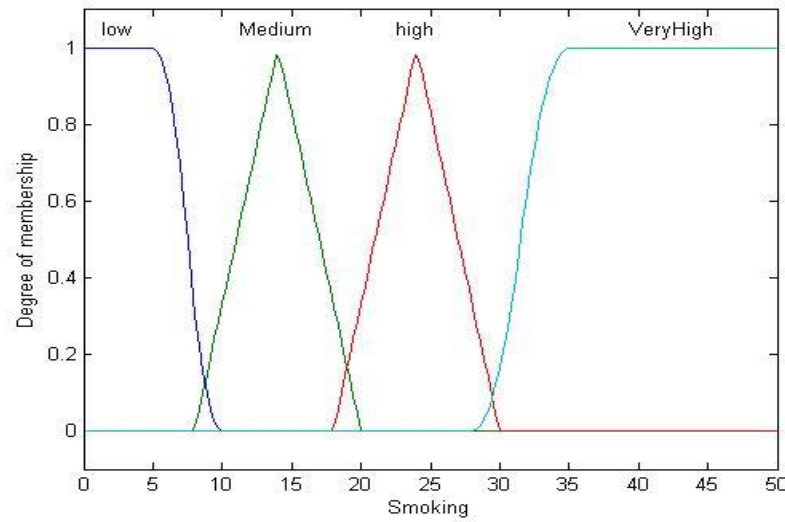

Figure10: Linguistic variables and membership function of 'Smoking'.

\subsection{Output variable}

The output field refers to the percentage of Hypertension Risk in the patient. It is classified in four classes; low, Mild, Moderate and Severe. As this percentage increases, Hypertension risk factor increases. We have considered trapezoidal membership functions for analysis and these are mentioned in following figure.

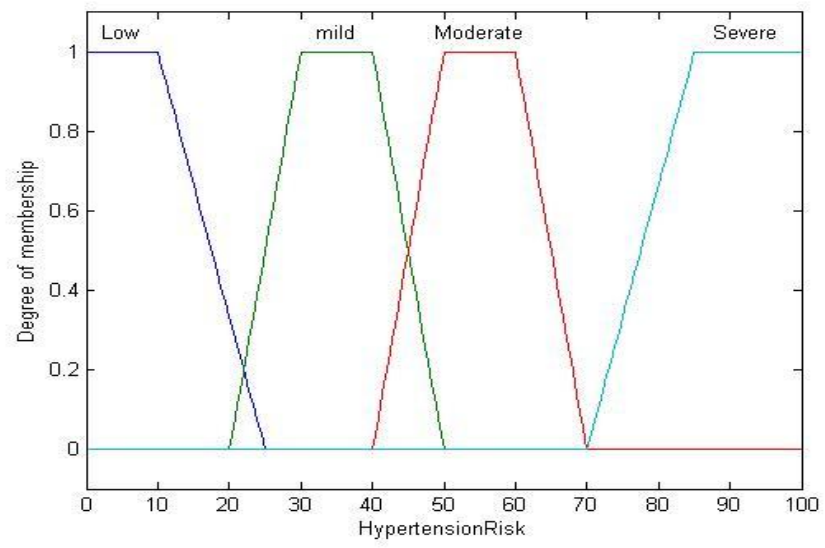

Figure11: Linguistic variables and membership function of output 'Risk'.

\subsection{Fuzzy rule}

The Rule Base consists of a set of Fuzzy compositions and is derived from the Knowledge Base of the Medical Experts. A fuzzy statement establishes a relationship between different input fuzzy sets and output sets. Some of the rules are given below in table.

\begin{tabular}{lllllllllll}
\hline & & & \multicolumn{9}{c}{ Input Variables } & & & & Output \\
variable
\end{tabular}




\begin{tabular}{|c|c|c|c|c|c|c|c|c|c|c|}
\hline Aged & low & normal & borderline & normal & Ab. nor & borderline & normal & 6 & $45 \mathrm{~min}$ & low \\
\hline ----- & ------ & ------- & ------ & ------ & ------ & ------ & ------- & ------ & ------ & ------ \\
\hline Aged & medium & normal & high & Mod. & Mod. & normal & high & 15 & no & mild \\
\hline---- & ------ & ------- & ------ & ------ & ------ & ------ & ------- & ------ & ------ & ------ \\
\hline Aged & medium & high & high & Mod. & Mod. & high & high & 2 & $60 \mathrm{~min}$ & moderate \\
\hline Aged & high & high & high & high & high & borderline & high & 12 & no & severe \\
\hline---- & ------ & ------- & ------ & ------ & ------ & ------ & ------- & ------ & ------ & ------ \\
\hline old & medium & normal & normal & Ab. nor & normal & borderline & normal & 2 & $20 \mathrm{~min}$ & low \\
\hline ----. & ------ & -------- & ------ & ------ & ------ & ------ & -------- & ------ & ------ & ------ \\
\hline old & medium & high & borderline & LH & LH & borderline & high & 8 & $30 \mathrm{~min}$ & mild \\
\hline ---- & ------ & ------- & ------ & ------ & ------ & ------ & ------- & ------ & ------ & ------ \\
\hline old & medium & high & borderline & high & LH & high & normal & 7 & $60 \mathrm{~min}$ & moderate \\
\hline ----- & ------ & ------- & ------ & ------ & ------ & ------ & ------- & ------ & ------ & ------ \\
\hline $\begin{array}{l}\text { Very } \\
\text { old }\end{array}$ & high & high & high & high & high & high & normal & 4 & $90 \mathrm{~min}$ & severe \\
\hline $\begin{array}{l}\text { Very } \\
\text { old }\end{array}$ & $\begin{array}{l}\text { Very } \\
\text { high }\end{array}$ & Very high & Very high & $\begin{array}{l}\text { Very } \\
\text { high }\end{array}$ & $\begin{array}{l}\text { Very } \\
\text { high }\end{array}$ & Very high & $\begin{array}{l}\text { Very } \\
\text { high }\end{array}$ & 12 & $60 \mathrm{~min}$ & severe \\
\hline
\end{tabular}

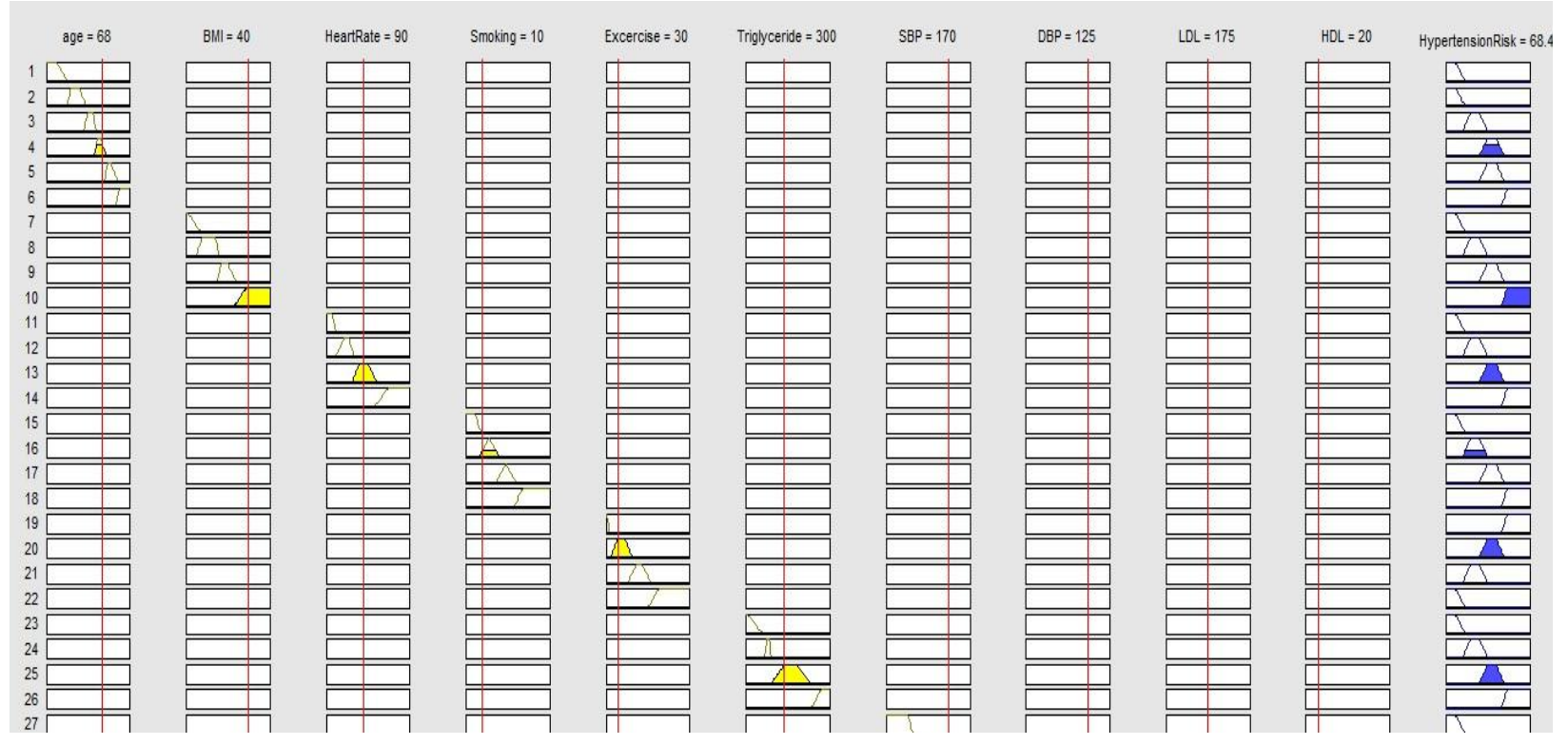

Figure12: Result of FES

\section{DEFUZZIFICATION}

The main objective of this study is to determine hypertension risk based on the linguistics description of the input parameters Age, SBP, DBP, BMI, LDL, HDL, Triglyceride, Heart rate, smoking and exercise. Hypertension risk will be assessed by different antecedent parts but with the same consequence.

\section{RESULT}

The rules have been developed using if- then method. Figure 13 shows result for person of age 68 , BMI of $40 \mathrm{~kg} / \mathrm{m} 2$, heart rate 90 beats $/ \mathrm{min}$, Triglyceride $300 \mathrm{mg} / \mathrm{dL}$, SBP $170 \mathrm{~mm}$, DBP $125 \mathrm{~mm}, \quad$ LDL $175 \mathrm{mg} / \mathrm{dL}$ and HDL $20 \mathrm{mg} / \mathrm{dL}$ having hypertension risk of about $69 \%$. The figure 13 (b) shows 3D surface diagram in between HDL and LDL. It is very much clear that, at low LDL and Low HDL hypertension risk is moderate and for increasing value of HDL, Hypertension risk will decrease. As a consequence, we may say that higher HDL and Lower LDL level decreases risk for Hypertension and Heart disease.

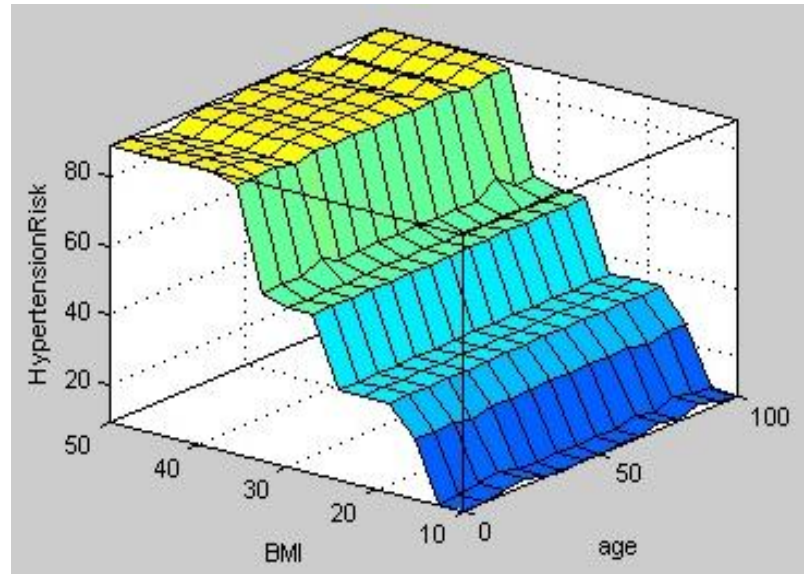

Fig 13: (a) 


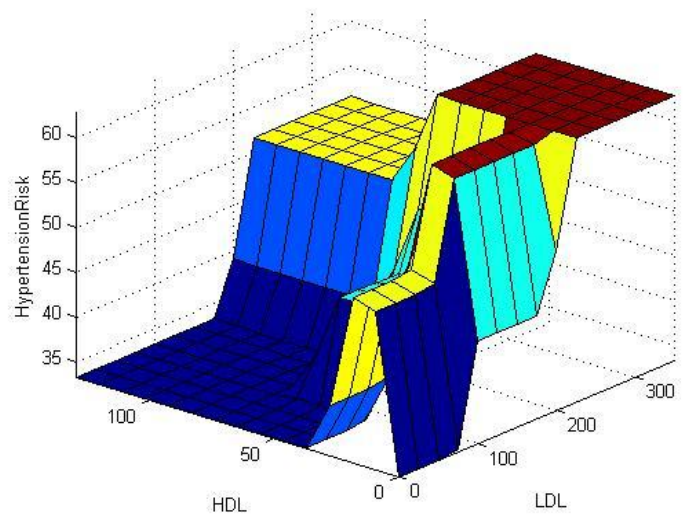

Fig 13(b)

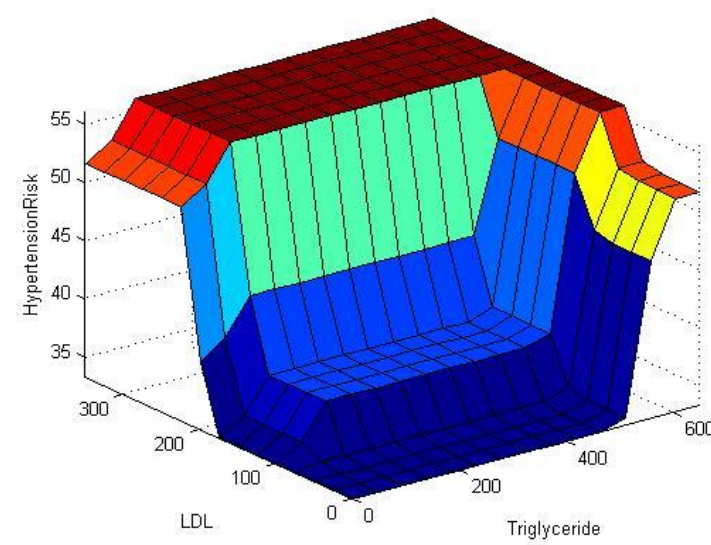

Fig 13 (c)

Figure13: (a), (b), (c) Surface view of FES.

\section{CONCLUSION}

The diagnosis of hypertension involves several layers of uncertainty and imprecision. The task of hypertension diagnosis and management is complex because of the numerous variables involved. Patients cannot describe exactly how they feel; doctors and nurses cannot tell exactly what they observe. The present research article confirms that the fuzzy expert system can represent the expert's thinking in a satisfactory manner in handling complex cases. The proposed FES is user friendly and effective for hypertension management and it has been verified by medical experts.

\section{REFERENCES}

[1] Degoulet P., Devries C., and Sauquet D., 1989 "Data and knowledge management integration in hospital information system," in Towards News Information Systems, Amsterdem,North-Holland, pp. 149-156.

[2] Szolovits, P., Patil, R.S., and Schwartz, W.B. 1988. "Artificial Intelligent in Medical Diagnosis".Journal of Internal Medicine. 108(1):80-87.

[3] Hobbs, R. and Boyle, A. 2004. Disease Management Project. ACC/AHA Guidelines for the Evaluation and Management of Chronic Heart Failure in the Audit: Executive Summary. American College of Cardiology/American Heart Association: Washington, D.C.

[4] Djam X.Y. and Kimbi Y.H., 2011. "Fuzzy Expert System for the Management of Hypertension" The Pacific Journal of Science and Technology Volume 12. Number 1.May 2011 (Spring)
[5] Riccardo P. and Stefano C. 1991 “A Neural Network Expert Systemfor Diagnosing and Treating Hypertension" University of Florence Riccardo Livi, pp.64-71.

[6] Sylvie C., Sylvie G., Gilles M., and Jean P. S.,2000 "Statistical and Fuzzy Models of Ambulatory Systolic Blood Pressure for Hypertension Diagnosis" IEEE Transactions on Instrumentation And Measurement, Vol. 49, No. 5, pp.998-1003

[7] Novruz ALLAHVERDI, Serhat and TORUN Ismail SARITAS, 2007 "Fuzzy Expert System Design for Determination of Coronary Heart Disease Risk" International Conference on Computer Systems and Technologies .

[8] Pandey D., Mahajan Vaishali \& Srivastava Pankaj 2006 "Rule Based System for Cardiac Analysis", NATL ACAD SCI LETT, Vol. 29, No. 7\&8, pp 299-309

[9] Srivastava Pankaj, Srivastava Amit 2012 "A Soft Computing Approach for Cardiac Analysis" Journal of Basic and Applied Scientific Research, 2(1)376-385

[10] Chen, C.L. and Chen, W.C. 1994. "Fuzzy Controller Design by Using Neural Network Techniques". IEEE Transactions on Fuzzy Systems. 2(3):235-244.

[11] Nalayini, N. and Wahidabanu, R.S.D. 2008. "Design Methodology of a Controller to Forecast the Uncertain Cardiac Arrest Using Fuzzy Logic Approach". International Conference Intelligent Systems and Controls: Karpagam College of Engineering, Coimbatore. 46-52. 